\title{
Detection of genioglossus myoelectric activity using ICA of multi-channel mandible sEMG
}

\author{
Tianyi Song ${ }^{\mathrm{a}, \mathrm{b}, 1}$, Baowen Meng ${ }^{\mathrm{a}, 1}$, Baoming Chen ${ }^{\mathrm{c}}$, Di Zhao ${ }^{\mathrm{d}}$, Zhengtao Cao $^{\mathrm{e}}$, \\ Jingying Ye $\mathrm{Y}^{\mathrm{d}, *}$ and Mengsun $\mathrm{Yu}^{\mathrm{b}, \mathrm{e}, *}$ \\ ${ }^{a}$ School of Biomedical Engineering, Fourth Military Medical University, Xi'an, Shaanxi, China \\ ${ }^{\mathrm{b}}$ Department of Medical Engineering, General Hospital of Jinan Military Command, Jinan, Shandong, \\ China \\ ${ }^{\mathrm{C}}$ School of Physics and Electronic Engineering, Shanxi University, Taiyuan, Shanxi, China \\ ${ }^{\mathrm{d}}$ Department of Otolaryngology, Head and Neck Surgery, Beijing Tongren Hospital, Capital Medical \\ University, Beijing, China \\ ${ }^{\mathrm{e}}$ Center of Aviation Medical Engineering, Institute of Aviation Medicine Beijing, Beijing, China
}

\begin{abstract}
.
BACKGROUND: Genioglossus myoelectric activity is of great significance in evaluating clinical respiratory function. However, there is a tradeoff in genioglossus EMG measurement with respect to accuracy versus convenience.

OBJECTIVE: This paper presents a way to separate the characteristics of genioglossus myoelectric activity from multi-channel mandible sEMG through independent component analysis.

METHODS: First, intra-oral genioglossus EMGgenioglossus EMG and three-channel mandible sEMG were recorded simultaneously. The FastICA algorithm was applied to three independent components from the sEMG signals. Then the independent components with the intra-oral genioglossus EMG were compared by calculating the Pearson correlation coefficient between them.

RESULTS: An examination of 60 EMG samples showed that the FastICA algorithm was effective in separating the characteristics of genioglossus myoelectric activity from multi-channel mandible sEMG. The results of analysis were coincident with clinical diagnosis through intra-oral electrodes.

CONCLUSIONS: Genioglossus myoelectric activity can be evaluated accurately by multi-channel mandible sEMG, which is non-invasive and easy to record.
\end{abstract}

Keywords: Electromyography, genioglossus muscle, independent component analysis

\section{Introduction}

The genioglossus (GG) muscles are a pair of fan-shaped muscles found on either side of the tongue's midline; they extend from the mandible backward and upward. As GG muscles are important pharyngeal

\footnotetext{
${ }^{1}$ Song Tianyi and Meng Baowen contributed equally to this study and share first authorship.

${ }^{*}$ Corresponding author: Mengsun Yu, Center of Aviation Medical Engineering, Institute of Aviation Medicine Beijing, Beijing, China. Tel.: +86 106692 7047; Fax: +86 106845 3059; E-mail: yms1601 @ gmail.com. Jingying Ye, Department of Otolaryngology, Head and Neck Surgery, Beijing Tongren Hospital, Capital Medical University, Beijing, China. Tel.: +86 10 56118899; Fax: +86 10 56118500; E-mail: tryejy@ gmail.com.
} 
dilators that keep the upper airway open, they are of great significance in evaluating clinically respiratory function [1]. The measurement of GG electromyography (EMG) has caught widespread attention. Many researchers have tried to record GG EMGs in various ways. Some inserted percutaneous finewire electrodes [2-4], hook-wire electrodes [5], or concentric needle electrodes [6] into the GG; others placed intra-oral surface electrodes [7]8] on the GG. These two methods are generally considered accurate. However, the invasive or intra-oral electrodes used are uncomfortable, especially during long-term measurement. Accordingly, some researchers have placed a pair of surface electrodes on the mandible, which are near the GG, and recorded mandible surface electromyography (sEMG) more comfortably [9]. Howver, these signals come not only from the GG, but also from neighboring muscle groups, and therefore cannot represent GG myoelectric activity.

Independent component analysis (ICA) is an important method for blind source separation and has been successfully applied to separate mixed signals when the number of active sources is unknown [10]. According to statistics independent rules, ICA can decompose the original mixtures to independent components (ICs). In recent years, ICA has been proved suitable for separating myoelectric signals. Using ICA, the eight-channel sEMG signals recorded from the tibial anterior muscles have been decomposed successfully to motor unit action potentials [11-13]. Some researchers have used ICA of sEMG to identify complex hand and finger movements [14|15]. So far, there are few reports on application of ICA in analysis of the GG EMG.

Since sEMG measures the electrical activity of a number of neighboring muscles [10], the mandible sEMG signals come from EMG generated by neighborhood muscle groups, including the GG. Therefore, ICA processing can separate GG EMG from the mandible sEMG signals; thus the GG EMG can be recorded by electrodes mounted on the mandible. This is non-invasive, accurate, and more patientfriendly. Based on the ideas above, this research simultaneously recorded three-channel mandible sEMG signals and intra-oral GG EMG, processed the former with ICA, and then compared the decomposed ICs with the latter by calculating the Pearson correlation coefficient between them and using statistical analysis.

\section{Materials and methods}

\subsection{Subjects}

Six healthy people aged 25-32 years, including two males and four females, were recruited for the study. These people suffered from oral cavity disease, sleep apnea syndrome, or correlative diseases. The Ethics Committee of Beijing Tongren Hospital approved the study, and each subject gave informed consent.

\subsection{EMG electrodes}

Silver-silver-chloride $(\mathrm{Ag}-\mathrm{AgCl})$ surface electrodes (diameter, $6 \mathrm{~mm}$ ) were used to record the mandible sEMG signals for their favorable impedance characteristics. The electrodes were mounted on the mandible by conductive gel and fabric. Figure 1 shows the position of the mandible sEMG electrode array. The reference electrode No. 0 was located approximately $10 \mathrm{~mm}$ away from the mandibular incisal edge, under which there was an electrode array, including electrode No. 1 to No. 4.

A customized dental plate of the mandibular teeth and the mouth floor was made for every subject, and a pair of $\mathrm{Ag}-\mathrm{AgCl}$ globular electrodes (diameter, $4 \mathrm{~mm}$ ) were mounted under the plate (Fig. 2). 


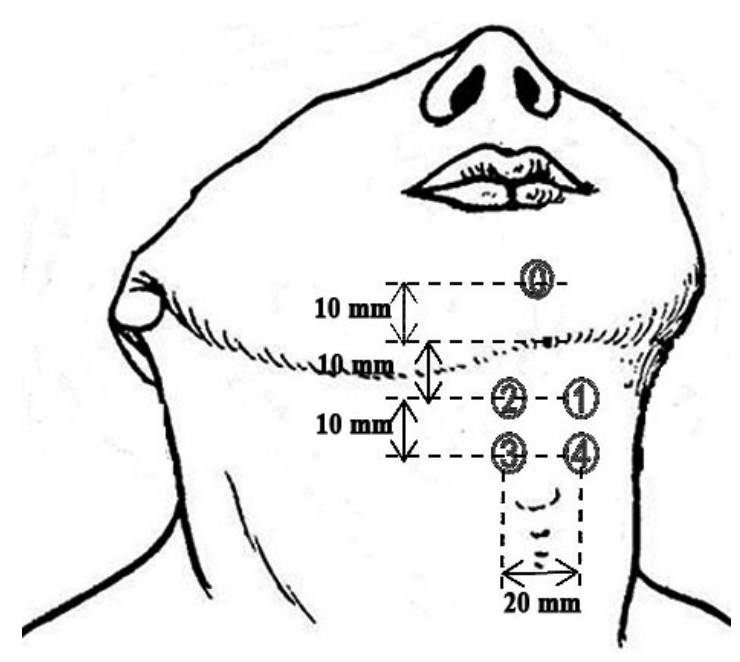

Fig. 1. Schematic diagram of mandible sEMG electrode array.

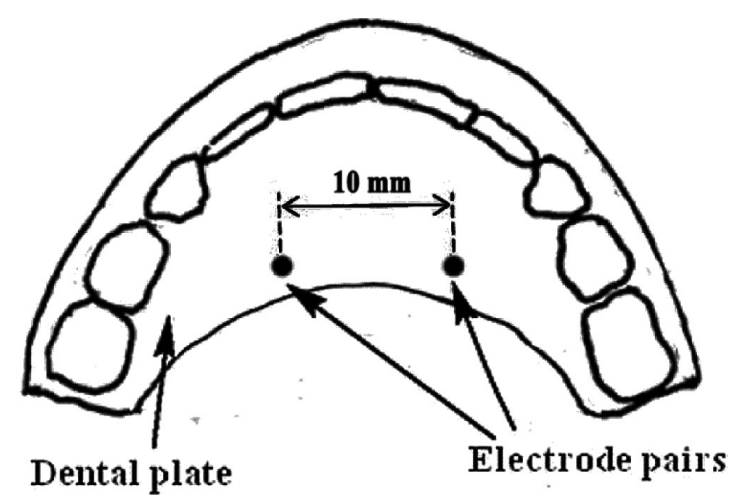

Fig. 2. Schematic diagram of intra-oral electrode pairs for GGm EMG.

The distance between the electrode pairs was about $10 \mathrm{~mm}$. When the plate was placed in the subject's mouth and fitted securely against the teeth, the electrodes were in direct contact with the GG ridge. The intra-oral GG EMG were recorded with the electrode pairs.

\subsection{EMG signals acquisition}

The electrodes were connected to a four-channel bipolar analog amplifier with a magnification of 24 times. The mandible electrode No. 0 was connected to the reference terminal of the amplifier, electrodes No. 1-3 to the positive terminal of one channel, and electrode No. 4 to the negative terminals of the No. $4+$ channels. The intra-oral electrodes were connected to the fourth channel, with the left one to the positive terminal and the right to the negative terminal. Amplified signals were imported to a 24 bits sigma-delta mode analogue-to-digital converter with a sampling rate of 500 points per second. The system was battery-powered, and Bluetooth technology transported the digitized data to a computer; the common-mode interference was suppressed as much as possible.

\subsection{Experimental protocol}

The subjects were asked to keep lie prostrate and breathe regularly, swallow, and extend their tongue or mandible ten times separately. The mandible sEMG and the intra-oral GG EMG were recorded simultaneously.

\subsection{Grouping of signals}

The recorded signals were divided into segments under the guidance of an experienced sleep medicine specialist. Every segment corresponds to a movement in reference to the intra-oral GG EMG. The segments were assigned to four groups in accordance with the movement type: regular breathing, swallowing, tongue extension, and mandible extension. Every group consisted of 60 segments. 
Table 1

Comparison of maximum correlation coefficient of sEMGs-EMG $\mathrm{GG}_{\mathrm{G}}$ with that of ICs-EMG $\mathrm{GG}$

\begin{tabular}{lcccc}
\hline Groups & Sample cases & $\mathrm{r}(\mathrm{sEMGs-EMG} \mathrm{GG})(\bar{x} \pm s)$ & $\mathrm{r}\left(\mathrm{ICs}-\mathrm{EMG}_{\mathrm{GG}}\right)(\bar{x} \pm s)$ & $P$ \\
\hline Regularly breathing & 60 & $0.20 \pm 0.12$ & $0.33 \pm 0.14$ & 0.0653 \\
Swallowing & 60 & $0.26 \pm 0.08$ & $0.59 \pm 0.16$ & 0.0322 \\
Tongue extension & 60 & $0.23 \pm 0.11$ & $0.58 \pm 0.23$ & 0.0276 \\
Mandible extension & 60 & $0.30 \pm 0.14$ & $0.62 \pm 0.19$ & 0.0227 \\
\hline
\end{tabular}

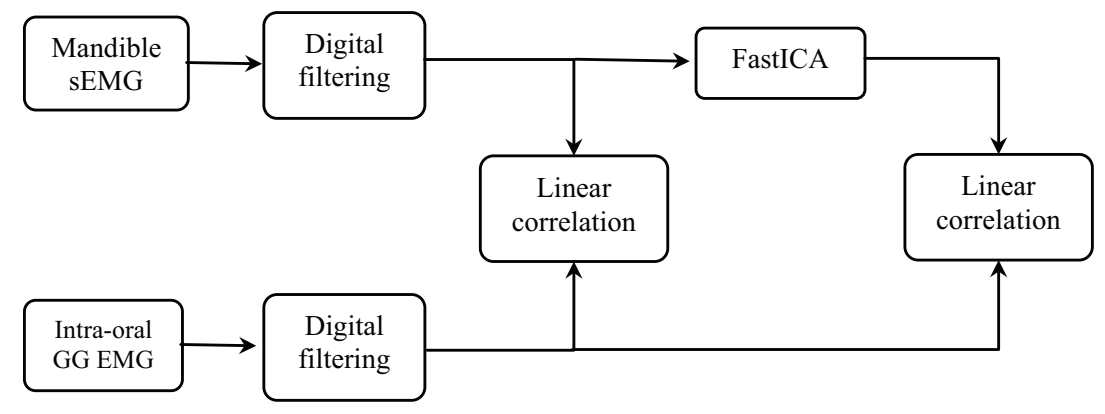

Fig. 3. The flowchart of the proposed procedure for EMG signals.

\subsection{EMG signals processing}

Figure 3 shows the flowchart of the proposed procedure for the EMG signals. First, the mandible sEMG and the intra-oral GG EMG were preprocessed by a digital band-pass filtering whose passband is $10-100 \mathrm{~Hz}$.

ICA decomposed the preprocessed mandible sEMG signals to three ICs. The FastICA algorithm developed by Hyvärinen, which is a computationally efficient and robust fixed-point type algorithm [16], was applied. The FastICA package for MATLAB [16] was used to process the mandible sEMG data, and the hyperbolic tangent function was selected as the nonlinear function.

The Pearson correlation coefficient between every IC and the intra-oral GG EMG were separately calculated for each movement group; the maximum was assigned to the processing group. The Pearson correlation coefficient between every channel mandible sEMG and the intra-oral GG EMG was separately calculated; the maximum was assigned to a control group.

\subsection{Statistical analysis}

For each movement group, paired t-tests were used to compare the maximum Pearson correlation coefficient between the IC and the intra-oral GG EMG with the maximum Pearson correlation coefficient between the mandible sEMG and the intra-oral GG EMG; this represents how close the mandible sEMG signals are to the intra-oral GG EMG signal, before and after ICA processing.

Results are presented as the means \pm standard deviations. Data analysis was carried out with IBM SPSS 20 statistical package. Statistical significance was accepted at $P<0.05$.

\section{Results}

Table 1 compares the maximum correlation coefficient of $s E M G s-E_{G G}$ to that of ICs-EMG $\mathrm{GG}_{\mathrm{G}}$. For the regularly breathing group, there is no significant difference before and after ICA processing, 
but there is a significant difference before and after ICA processing for the other three groups. It can be concluded that the results of ICA processing were correlated to $\mathrm{EMG}_{\mathrm{GG}}$ for all groups except the regularly breathing group.

\section{Discussion}

Anatomical structure suggests that mandible sEMG signals are composed of EMG signals produced by neighboring muscle groups, including the GG, geniohyoid, mylohyoideus, stylohyoideus, etc. Therefore, the mandible sEMG cannot directly represent GG myoelectric activity. On the other hand, ICA processing can separate the GG EMG can be separated from the mandible sEMG signals.

ICA can find a linear representation of data so that the components are statistically independent or as independent as possible [16]. As the recruitment pattern of a muscle's motor units are not dependent on other muscles, and the motor unit action potentials (MUAP) do not overlap, each muscle can be assumed to be independent and non-Gaussian [15]. Therefore, ICA can decompose the mandible sEMG signals to several independent sources, including the GG EMG.

In this study, the Pearson correlation coefficient was used to represent how close the sEMG signals were to the intra-oral GG EMG signal before and after ICA processing. As is indicated by the results of statistical analysis, when the subjects exercised movements of swallowing, tongue extension, or mandible extension, the decomposed ICs were closer to the intra-oral GG EMG than the mandible sEMG, which represents GG myoelectric activity. However, the same conclusion cannot be verified when the subjects breathe regularly. This can be explained by the discharge regularity of GG muscles [2]. During regular breathing, GG myoelectric activity was so weak that the discharge signals were overwhelmed by disturbance.

Since the ICA treated both disturbance and useful signals equally, it is necessary to enhance the signalto-noise ratio of recorded signals. In this study, a highly accurate 24 bits sigma-delta mode analogueto-digital converter was used to record the weak EMG signals, and the signal acquisition system was battery-powered to guarantee quality of the signals. On the other hand, ICA is suitable when the numbers of recordings are the same as or greater than the number of sources [10]; the quantity of input channels can affect the reliability of ICA. Electrode configurations were considered carefully for the surface area of electrodes and the limited amount of channels, and three-channel bipolar signals were acquired subtly from only four electrodes. The steps mentioned above contributed to the effectiveness of ICA processing.

\section{Conclusion}

GG EMG provides important information about GG myoelectric activity, and present measurement methods are unsatisfactory. Intramuscular or intra-oral electrodes make patients uncomfortable and cannot be used for long-term clinical monitoring. While mandible electrodes are not accurate and difficult to identify the respiratory events effectively.

This paper presented a novel method to record GG EMG. Three-channel mandible sEMG signals were recorded with more mandible electrodes and a highly accurate analogue-to-digital converter. Then the FastICA algorithm was used to decompose the sEMG signals to three ICs. An experiment was conducted to compare the ICs with the intra-oral GG EMG signals recorded simultaneously. The results indicate that ICs are consistent with the intra-oral GG EMG during the subjects' movements of swallowing, tongue extension, or mandible extension. Compared with existing methods, the method is non-invasive, 
accurate, and can be valuable for clinical application. In the future, the authors would like to customize tiny electrodes to obtain multi-channel EMG signals and improve the amount of ICA input channels. More subjects may be recruited, and long-term monitoring will be conducted to improve and verify the proposed method.

\section{Acknowledgement}

This work is supported by Key Technologies Research and the Development Program of China \#2013BAI03B05.

\section{References}

[1] McSharry DG, Saboisky JP, Deyoung P, Matteis P, Jordan AS, Trinder J et al. A mechanism for upper airway stability during slow wave sleep. Sleep. 2013; 36(4): 555-63. doi: 10.5665/sleep.2544.

[2] Wilkinson V, Malhotra A, Nicholas CL, Worsnop C, Jordan AS, Butler JE et al. Discharge patterns of human genioglossus motor units during arousal from sleep. Sleep. 2010; 33(3): 379-87.

[3] Nicholas CL, Bei B, Worsnop C, Malhotra A, Jordan AS, Saboisky JP et al. Motor unit recruitment in human genioglossus muscle in response to hypercapnia. Sleep. 2010; 33(11): 1529-38.

[4] Eastwood PR, Allison GT, Shepherd KL, Szollosi I, Hillman DR. Heterogeneous activity of the human genioglossus muscle assessed by multiple bipolar fine-wire electrodes. J Appl Physiol (1985). 2003; 94(5): 1849-58. doi: 10.1152/japplphysiol.01017.2002.

[5] Pittman LJ, Bailey EF. Genioglossus and intrinsic electromyographic activities in impeded and unimpeded protrusion tasks. J Neurophysiol. 2009; 101(1): 276-82. doi: 10.1152/jn.91065.2008.

[6] Saboisky JP, Stashuk DW, Hamilton-Wright A, Carusona AL, Campana LM, Trinder J et al. Neurogenic changes in the upper airway of patients with obstructive sleep apnea. Am J Respir Crit Care Med. 2012; 185(3): 322-9. doi: 10.1164/ rccm.201106-1058OC.

[7] Doble EA, Leiter JC, Knuth SL, Daubenspeck JA, Bartlett DJ. A noninvasive intraoral electromyographic electrode for genioglossus muscle. J Appl Physiol. 1985; 58(4): 1378-82.

[8] O'Connor CM, Lowery MM, Doherty LS, McHugh M, O'Muircheartaigh C, Cullen J et al. Improved surface EMG electrode for measuring genioglossus muscle activity. Respir Physiol Neurobiol. 2007; 159(1): 55-67. doi: 10.1016/j.resp. 2007.05.011.

[9] Sauerland EK, Sauerland BA, Orr WC, Hairston LE. Non-invasive electromyography of human genioglossal (tongue) activity. Electromyogr Clin Neurophysiol. 1981; 21(2-3): 279-86.

[10] Naik GR, Kumar DK. Estimation of independent and dependent components of non-invasive EMG using fast ICA: validation in recognising complex gestures. Comput Methods Biomech Biomed Engin. 2011; 14(12): 1105-11. doi: 10. 1080/10255842.2010.515211.

[11] Ren X, Wang Z, Hu X. Independent component analysis and wavelet decomposition technique for the detection of motor unit action potentials. Conf Proc IEEE Eng Med Biol Soc. 2005; 3: 2687-90. doi: 10.1109/IEMBS.2005.1617024.

[12] Nakamura H, Yoshida M, Kotani M, Akazawa K, Moritani T. The application of independent component analysis to the multi-channel surface electromyographic signals for separation of motor unit action potential trains: part I-measuring techniques. J Electromyogr Kinesiol. 2004; 14(4): 423-32. doi: 10.1016/j.jelekin.2004.01.004.

[13] Nakamura H, Yoshida M, Kotani M, Akazawa K, Moritani T. The application of independent component analysis to the multi-channel surface electromyographic signals for separation of motor unit action potential trains: part II-modelling interpretation. J Electromyogr Kinesiol. 2004; 14(4): 433-41. doi: 10.1016/j.jelekin.2004.01.005.

[14] Naik GR, Acharyya A, Nguyen HT, "editors'. Classification of finger extension and flexion of EMG and Cyberglove data with modified ICA weight matrix. Engineering in Medicine and Biology Society (EMBC), 2014 36th Annual International Conference of the IEEE; 2014 2014-01-01; Chicago, IL.

[15] Naik GR, Kumar DK. Identification of hand and finger movements using multi run ICA of surface electromyogram. J Med Syst. 2012; 36(2): 841-51. doi: 10.1007/s10916-010-9548-2.

[16] Bingham E, Hyvarinen A. A fast fixed-point algorithm for independent component analysis of complex valued signals. Int J Neural Syst. 2000; 10(1): 1-8. doi: 10.1142/S0129065700000028. 